\title{
Telepresence Robot Helps Children in Communicating with Teachers who Speak a Different Language
}

\author{
Fumihide Tanaka \\ The University of Tokyo \\ 7-3-1 Hongo, Bunkyo-ku \\ Tokyo 113-8656, Japan \\ fumihide.tanaka@gmail.com \\ Nao Tazawa \\ University of Tsukuba \\ 1-1-1 Tennodai, Tsukuba-shi \\ Ibaraki 305-8573, Japan \\ s1320839@u.tsukuba.ac.jp
}

Toshimitsu Takahashi

University of Tsukuba

1-1-1 Tennodai, Tsukuba-shi

Ibaraki 305-8573, Japan

10432.t@gmail.com

\author{
Shizuko Matsuzoe \\ University of Tsukuba \\ 1-1-1 Tennodai, Tsukuba-shi \\ Ibaraki 305-8573, Japan \\ shi25tty@gmail.com
}

\author{
Masahiko Morita \\ University of Tsukuba \\ 1-1-1 Tennodai, Tsukuba-shi \\ Ibaraki 305-8573, Japan \\ mor@bcl.esys.tsukuba.ac.jp
}

\begin{abstract}
This study reports the advantages of using a child-operated telepresence robot system for the purpose of remote education. Video conferencing is already common in educational settings, where a foreign language is taught by a native teacher from a remote location; however, there is a serious issue in that children tend to have difficulties or freeze when facing teachers who speak a different language over a monitor. We hypothesize that a child-operated telepresence robot that offers physical participation and operability will help to address this issue. To investigate this hypothesis, we conduct a field experiment with 52 participants (4-8 years old) in classroom environments, and the use of a telepresence robot system is compared with a baseline Skype condition. The results show the advantages of the telepresence robot system for both children and teachers.
\end{abstract}

\section{Categories and Subject Descriptors}

I.2.9 [Artificial Intelligence]: Robotics; H.4.3 [Information Systems Applications]: Communications Applications-Computer conferencing, teleconferencing, and videoconferencing

\section{General Terms}

Design, Human Factors, Verification

\section{Keywords}

Telepresence robot, telerobotics, distant education, remote communication, children, video conferencing, foreign language teaching, communication difficulties
Permission to make digital or hard copies of all or part of this work for personal or classroom use is granted without fee provided that copies are not made or distributed for profit or commercial advantage and that copies bear this notice and the full citation on the first page. Copyrights for components of this work owned by others than ACM must be honored. Abstracting with credit is permitted. To copy otherwise, or republish, to post on servers or to redistribute to lists, requires prior specific permission and/or a fee. Request permissions from permissions@ acm.org. HRI'14, March 3-6, 2014, Bielefeld, Germany.

Copyright 2014 ACM 978-1-4503-2658-2/14/03 ...\$15.00.

http://dx.doi.org/10.1145/2559636.2559654.

\section{INTRODUCTION}

With the increasing popularity of video conferencing, many educational organizations have considered its use for the purpose of remote education. In addition to one-to-many lecture-style settings, it is also employed in one-to-one settings. An example of such a oneto-one setting is the teaching of foreign languages by native speakers who give lessons from distant locations. In fact, in countries such as Japan, there is a big demand for foreign language education by native speakers, and many language schools are introducing video conferencing in their lessons. However, classroom teachers often complain that video conferencing may not be ideal for teaching young children because they easily encounter difficulties and freeze when facing a teacher who speaks a different language over the monitor. As discussed in previous studies [1,2], the use of video conferencing provides children with very limited communication options. Teachers have also reported experiencing some difficulty, which was observed from their behavior when using video conferencing to teach children [3].

The use of telepresence robots may provide a useful solution for the above-explained issue. In contrast with standard video conferencing, where remote communication involves only audio and video, telepresence robots enable operators to physically interact with remote environments that include persons. Because physical interactions, such as handing objects to each other, play an important role in classroom activities involving young children, the use of telepresence robots could enable young children to communicate with teachers who speak a different language. This study explores this hypothesis by conducting a field experiment. The study is expected to become practically important from an educational perspective while providing a useful application for telepresence robots.

In Section 2, we begin by reviewing existing studies involving the use of telepresence robots for children. In fact, there are few studies involving telepresence robots that specifically target child users. When we started our initial project in 2009 , there had been no previous attempts for developing a telepresence robot system that is operable by young children. Then, we developed several prototypes of telepresence robots that were operable by young children including the one used for the experiment reported in this study (see Section 2). In Section 3, we briefly report about pilot observations we made in schools before the experiment started. In Section 4, we present the original contribution of this study, which describes 


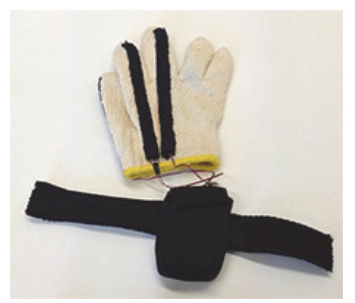

Figure 1: Data glove equipped with bending sensors extending along the fingers and a motion sensor that was attached on the wrist together with a Bluetooth circuit.

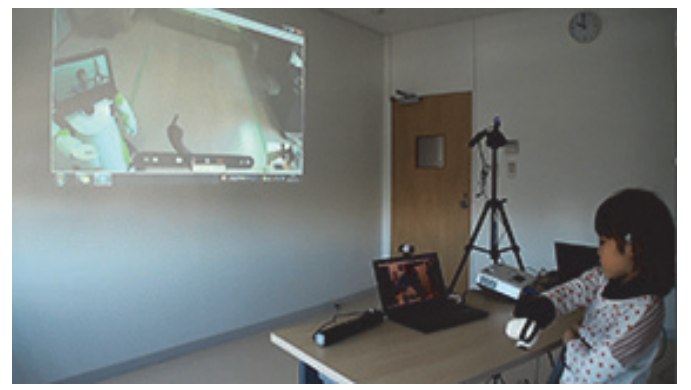

Figure 2: A child remotely controlling the telepresence robot using the data glove shown in Figure 1.

the field experiment mentioned in the previous sentence and the results obtained from the experiment. The experiment was conducted considering two conditions. In one condition, participants used only Skype monitors to join a lesson that was remotely conducted by a teacher who spoke a different language. For the other condition, participants were given both the Skype monitors and a remote-control interface for a telepresence robot that was placed in a room on the teacher's side. A total of 52 Japanese children (4-8 years old) participated in the experiment. The results (Section 4.3) show that the children's responses toward the teachers were significantly improved by the introduction of the remote-control interface. Furthermore, we found that the teachers also became more comfortable with the robot condition, and they were able to introduce a wider variety of teaching styles than with the baseline Skype condition. In Section 5, we discuss these results, and we provide a summary in Section 6.

\section{TELEPRESENCE ROBOT FOR CHILDREN}

Telepresence robots are gaining increased attention as new media for remote communication. Their application domain has been expanding, and companies have already commercialized telepresence robot products for use in areas such as healthcare, remote collaboration, and education $[4,5,6,7,8,9]$. In academia, studies have been conducted by the human-robot interaction (HRI) community to investigate the use cases and their potential impact on human users $[10,11]$.

Regarding the use of telepresence robots for educational purposes, one study discussed the development of a telepresence robot, which was remotely controlled by an adult teacher who gave lessons from a remote location using the robot [12]. The goal was to compensate for the shortage of native English teachers in South Korea. The research group developed a user interface for the teacher to remotely control the robot semi-autonomously. Also, a pilot

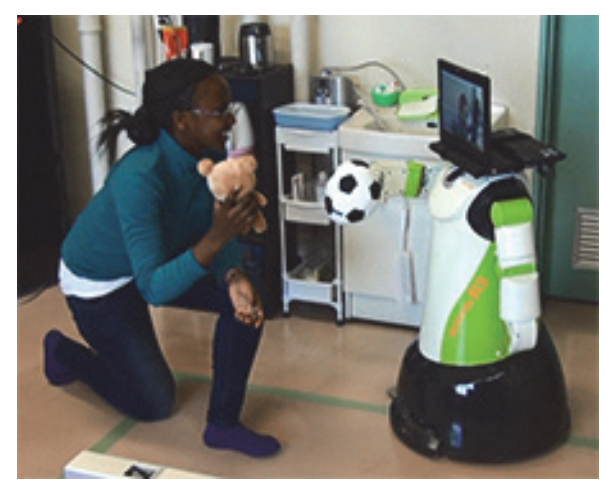

Figure 3: A teacher interacting with the telepresence robot that was remotely controlled by a child.

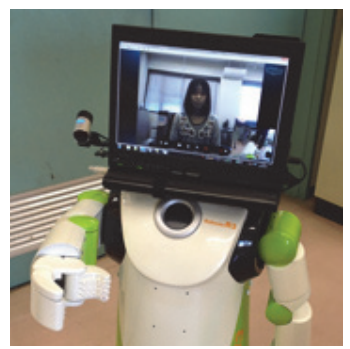

Figure 4: Teacher's view of the child operator on a Skype monitor mounted on the neck of the robot.

study was conducted in English classrooms, and positive feedback was received from teachers. In contrast, our previous study [3] attempted to develop a telepresence robot system that was remotely controlled by children. The main contribution of that study was the development of a tricycle-style operation interface, which enabled children as young as 3 years old to remotely control a robot placed in a remote location. Then, we also conducted an international field trial, which involved connecting two classrooms in Australia and Japan using the system [13]. As will be explained in Section 3, we observed that children enjoyed interacting with other children in foreign countries, and it appeared that the idea of using telepresence robots would work well for remote education. However, it remained unclear whether the introduction of a child-operated telepresence robot system would make a difference in remote education compared with standard video conferencing. Thus, we decided to perform additional studies in this area.

Considering the age range (4-8 years old) of participants for the experiment that will be described in Section 4, we improved the operation interface so that the operator could remotely control the movement of a telepresence robot using only one hand. Figure 1 shows the newly developed operation interface, which is a smallsized data glove equipped with a motion sensor (ZMP IMU-Z) with which the child operator can remotely control the movement of the robot base (forward, backward, left, and right) and the opening/closing of its gripper hand. Figures 2 and 3 are snapshots taken during the experiment. The telepresence robot (Figure 4) used in this experiment was based on Robovie-R3, which is commercially available from Vstone Co., Ltd. A tablet PC and a monitor camera were mounted on the neck of the robot to enable a video conferencing function. The right hand was fixed in the forward position, and its gripper was remotely controlled by the data glove mentioned above. The robot base could move in forward, backward, right, 
and left directions, and it was remotely controlled by detecting the gestures of the participants, as explained above. Control signals sent from the operation interface were routed through a server PC and delivered to the telepresence robot. All of these signals were encrypted using SSH tunneling.

\section{PILOT OBSERVATIONS IN SCHOOLS}

In 2011 and 2012, we paid regular visits to an English learning school for Japanese children and four public classrooms in an elementary school and kindergartens in Tsukuba city, Japan. From discussions with teachers at those schools, we confirmed the importance of helping children at English lessons given by video conferencing. We also tried a pilot trial in which we asked four children to each play with a foreign teacher over a Skype monitor. Then, we found that with the exception of one child who had previously experienced living abroad, most of the children got stuck early in communication with the teacher, and eventually they were unable to do anything in front of the monitor. This confirmed what we had previously heard about the use of video conferencing for teaching young children.

We were also simultaneously working on another project of connecting two classrooms in Australia and Japan using a telepresence robot system. Although the goal was to provide an opportunity for international exchange by allowing children on both sides to interact with each other, we observed that even shy children could enjoy communicating with others on the remote side when a different language was used [13]. However, the participants of this international trial were older than our target children described above. Therefore, we modified the operation interface of the telepresence robot system, as explained in Section 2. Together with the feedback and suggestions received from the school teachers, we prepared the experiment that is explained in Section 4 below.

\section{EXPERIMENTS}

\subsection{Goal}

This experiment explores the effect of introducing a telepresence robot system into a remote English conversation involving Japanese children. In particular, we investigated the effect by comparing the system with a controlled condition in which a standard video conferencing technology (Skype) was used, which is a style that is commonly embraced by many English conversation schools in Japan. It is known that children often get stuck and freeze in front of teachers who speak only English while giving video conferencing lessons. We hypothesized that by introducing the telepresence robot system, children would be better equipped to interact with their teachers, and the teachers would also find it easier to give their lessons compared with using only video conferencing. The experiment was designed to explore this hypothesis.

\subsection{Method}

\subsubsection{Participants and Apparatus}

The experiment was conducted by connecting two rooms via the Internet. One room (Figure 3) was located at the University of Tsukuba, from where teachers gave English lessons to children in remote locations. A telepresence robot (Figures 3 and 4) was placed in the room, and the children remotely controlled the robot, as explained below. The other room was located at either an elementary school (Kasuga Elementary School) or at two kindergartens (Sakura Kindergarten and Higashi Kindergarten) in Tsukuba city, Japan, from where children operators (one child at a time) remotely controlled the robot. The experimental settings of these

\begin{tabular}{|c|c|c|}
\hline Skype-only condition & Skype & Skype \\
\hline Skype-robot condition & Skype & Robot \\
\hline
\end{tabular}

Figure 5: Between-participants design: participants were divided into either of the two condition groups.

three locations were setup such that they were as similar as possible. As seen in Figure 2, the operator was given two Skype monitors: one projected on the wall provided a bird's-eye view and the other on a laptop PC placed in front of the operator provided the view from a camera mounted on the neck of the telepresence robot. In the experimental condition in which the operator had access to remotely control the robot, the operator was given a control interface (Figure 1), which was explained in Section 2. Using the control interface, the operator could control the movement of the robot base (forward, backward, left, and right directions) and the opening/closing of the robot's right hand.

A total of 52 Japanese children (4-8 years old, 25 males and 27 females) participated in the experiment. After we received approval for this experiment from the Ethical Committee of the University of Tsukuba, we began to recruit participants by explaining and advertising our research goals to the parents of the children. After obtaining written consent from each parent, we started the experiment. Two experimenters were involved. Experimenter \#1, who was on the side of the children operators, gave an instruction to each of the children and maintained safety during the experiment. This individual also interviewed the children before and after each experimental session. Experimenter \#2, who was on the teacher side, gave instructions to the teachers and monitored the time during the experiment. The teachers were six native speakers of English at the University of Tsukuba. All of them were international students, and they were paid for helping with the experiment.

\subsubsection{Design}

We decided to adopt an experimental scenario in which each participant was asked to participate in a story-telling game (lesson) guided by a remote teacher who spoke only English. The lesson was given by video conferencing (Skype); however, half of the participants were provided with the remote-control interface (Figure 1) in the middle of the lesson with instructions about the telepresence robot placed on the teacher's side. This is an experiment using between-participants design, in which half of the participants were allocated a Skype-only condition, and the other half were allocated a Skype-robot condition (Figure 5). In this experiment, we decided to focus on an experimental variable, namely, the presence or absence of the control interface on the operator's side. The telepresence robot was always present on the teacher's side, and it moved only during the period in which the operator had access to the control interface. All of the teachers were deployed so that they experienced both of the conditions equally.

\subsubsection{Procedure}

Each experimental session involved one participant. At the beginning of the session, Experimenter\#1 took a short test to check the level of the participant's oral English. Experimenter \#1 greeted and talked to the participant in English for several minutes and observed the participant's reaction. Most of the participants (80$90 \%$ ) could not respond in English; however, there were some par- 


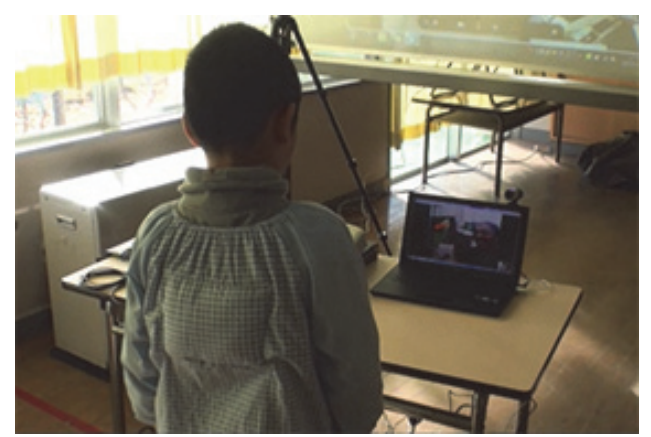

Figure 6: A participant completely stopping his movement (freezing) in front of Skype monitors: similar cases were frequently observed during the Skype period.

ticipants (10-20\%) who could respond in limited English. Then, Experimenter \#1 allocated the participant to either the Skype-only condition or the Skype-robot condition so that there was an almost equal distribution of participants for both conditions based on their levels of spoken English.

Each session lasted for $10 \mathrm{~min}$. If the participant belonged to the Skype-only condition group, he/she was told to join a storytelling game (lesson) guided by a native English teacher in a remote location using Skype monitors for $10 \mathrm{~min}$. On the other hand, if the participant belonged to the Skype-robot condition group, he/she was told to join the same lesson for 5 min using only the Skype monitors and then for another $5 \mathrm{~min}$ using the telepresence robot system (Figure 5). In the Skype-robot condition, Experimenter \#1 gave the participant the remote-control interface (Figure 1), along with instructions regarding its use when the first 5 min half ended.

The teacher conducted a story-telling conversation lesson in English from within a room located at the University of Tsukuba (Figure 3). At the beginning of each session, Experimenter \#2 gave three randomly chosen objects, e.g., stuffed animals, to the teacher. The teacher was instructed to improvise during the storytelling conversation lesson using the objects. The teacher was also instructed to use only English throughout the session, even if the participant did not understand the teacher's speech at all and got completely stuck.

Finally, at the end of the session, Experimenter \#1 performed a brief interview with the participant, asking about the experience of participating in the trial. The participants of the Skype-robot condition group were also asked about the type of lesson (Skype/Robot) that he/she preferred.

\subsection{Results}

\subsubsection{Behavioral Analyses of Children}

As expected, based on feedback received from professional teachers in private and public schools, in this experiment, we also encountered many situations in which participants completely stopped their movements in front of Skype monitors despite efforts made by the teacher in remote locations to encourage them to engage in the lesson (Figure 6). We quantified those situations by analyzing videos taken during the experiment. The first analysis was about the participants' responses toward the teacher. During the lesson, the teacher talked to each of the participants in various ways, and most of those actions were selected such that there was an expectation of some type of response from the participant. Using the videos, we extracted all of those actions using 14 human coders (coding reliability measurement: $\kappa=0.78$ ).

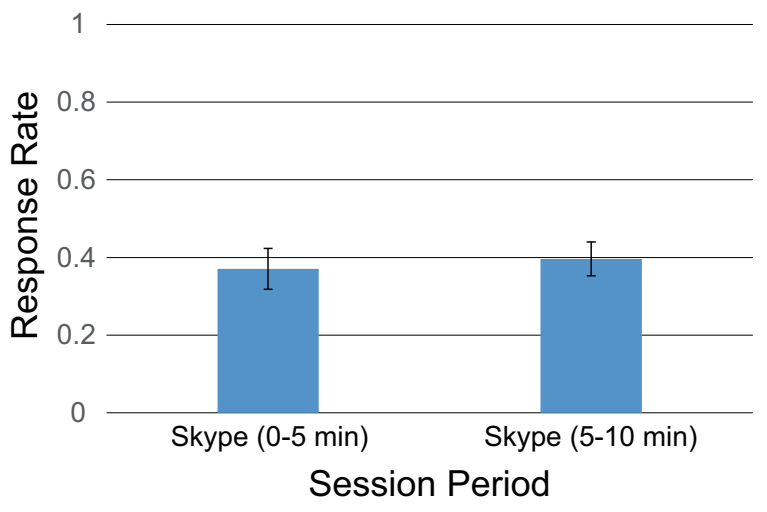

Figure 7: Average response rates of 24 participants allocated to the Skype-only condition toward the teacher's actions that were directed toward the participant. The rate did not change even if the teacher spent more time ( $>5 \mathrm{~min}$ ) focusing on teaching.

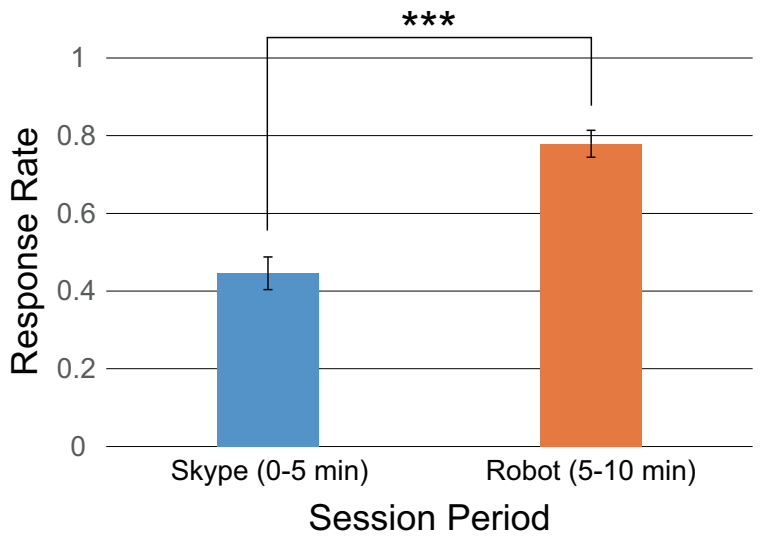

Figure 8: Average response rates of 28 participants allocated to the Skype-robot condition toward the teacher's actions that were directed toward the participant. The rate was significantly improved during the robot period (Wilcoxon signedrank test was conducted: $Z(28)=-4.60, p<0.001$ ).

As shown in Figure 7, the participants' response rates were consistently low in the Skype-only condition. Even if the teacher spent more time, which can be seen from the right bar (5-10 min), the average rate of the participants' responses did not change. In contrast, in the Skype-robot condition (Figure 8), at first, the response rate was nearly the same as that in the Skype-only condition; however, it then significantly improved for the robot period in which the participant was able to remotely control the telepresence robot.

In fact, we observed that many of the participants, even if they could not respond at all to the teacher in the Skype period, could move the robot and successfully communicate their intentions to the teacher during the robot period. Figure 9 shows an example. The participant on the left side of the pictures made almost no response toward the teacher during the first half of the session (the Skype period). However, in the latter half of the session (the robot period), the participant was able to follow each of the instructions given by the teacher (e.g., grabbing a banana and bringing it to a location), and he/she eventually spoke and learned the new word "banana." In general, a 5-min session appeared to be too short to enable all participants to reach this stage; however, for eight par- 

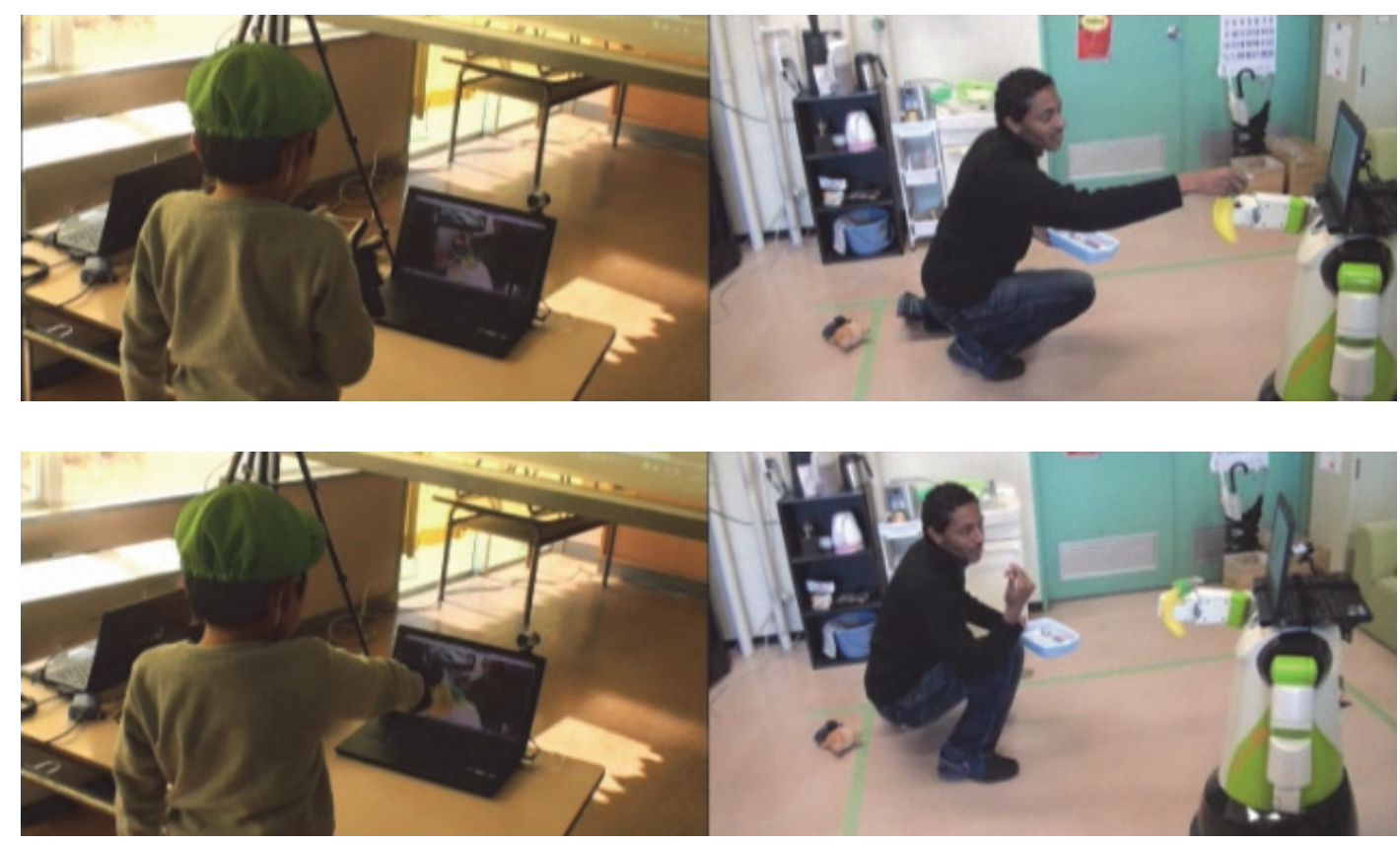

Figure 9: Example from the robot period: the participant on the left side of the images was grabbing a banana (top image) and then bringing it into a location (bottom image) while following the instructions of the teacher to the right side of the pictures. This participant made almost no response to the teacher during the Skype period. However, after interacting with the teacher by remotely controlling the robot, the participant spoke and learned words such as "banana," which was not observed during the Skype period.

ticipants, we confirmed that during the session, they were able to make clear utterances of newly learned English vocabulary. This was not observed during any of the Skype periods.

In addition to the positive changes observed in children during the robot period, teachers also became more comfortable in giving lessons in the robot period because they could better understand the intentions of the participant based on robot movement, and could thus create further teaching actions. We will discuss this point in detail in the next section.

\subsubsection{Behavioral Analyses of Teachers}

During the experiment, we noted that teachers also became more comfortable while giving their lessons during the robot period. One indication of this was that the teachers appeared to make positive utterances such as praising the participants many times during the robot period. To verify this, we conducted behavioral analyses on the teachers, as we did on the children, using videos taken during the experiment. Figures 10 and 11 show the results. Consistent with our initial impression, the results show a significant increase in the frequency of the teachers' positive utterances (such as praising the participant) during the robot period. It was also observed that in those situations, the participant understood the intention of the teacher and acted in a way desired by the teacher. The increase in the number of positive utterances was considered to be related to the satisfaction level of the teacher. Also, it means that the teacher was able to proceed to the next stage in the lesson, which is significantly different from the "stuck" situation that was frequently observed during the Skype period.

Next, we investigated the teaching styles of the teachers who participated in the experiment. By repeatedly observing the videos taken during the experiment, we found that the four categories listed below covered most of the teaching styles employed by the teachers:
One-way style: This was a style of teaching where the teacher talked to the participant unilaterally, i.e., continued to speak or explain something to the participant without prompting for any interaction such as asking him/her to answer questions.

Yes/No style: The teacher made an inquiry to the participant in a simple manner, asking yes or no.

5W1H style: The teacher made an inquiry to the participant in a manner using a $5 \mathrm{~W} 1 \mathrm{H}$ (when, where, who, what, why, and how) question.

Repetition style: The teacher made the participant repeat what the teacher was saying (e.g., "Say banana.")

The videos were coded by 14 human coders using these categories $(\kappa=0.83)$. Figure 12 shows the results obtained for the second Skype period (5-10 min) in the Skype-only condition. We also obtained the results from the first Skype period (0-5 min) for both the Skype-only and Skype-robot conditions, and those results were very similar to the one shown in Figure 12. As observed from the results, in the Skype period, the style that was most commonly used by teachers was the one-way style of teaching. However, after the experiment, many of the teachers told us that they had wanted to make the lessons more interactive, as opposed to mainly unilateral (one-way style), to attract the participants' interest. According to the teachers, this was not possible because of the unresponsiveness of the participants during the Skype period, and thus, the frequency with which the one-way style was used inevitably increased. In fact, some teachers had tried to use behaviors such as showing objects similarly to the way used during the robot period; however, it was not effective and then they eventually gave up (:these were considered as "Others" in Figure 12). In contrast, during the robot period, the use of the one-way style of teaching was significantly decreased (from $34 \%$ to $15 \%$ ), and styles of teaching that utilized 


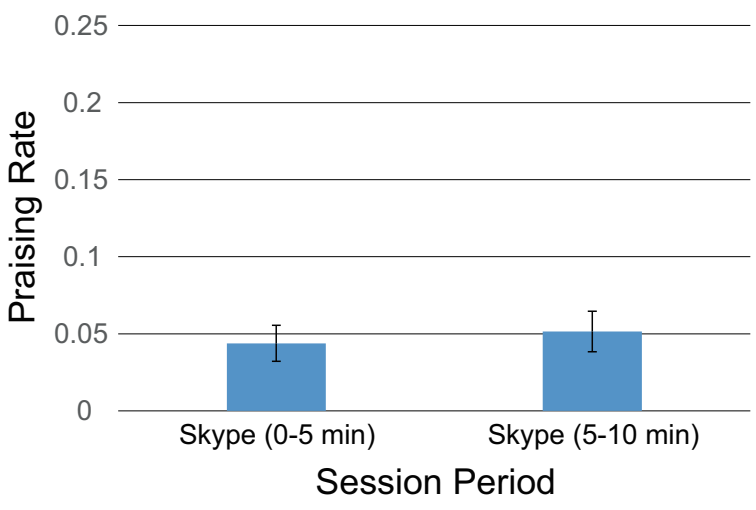

Figure 10: Average accumulated time during which teachers were making positive utterances such as praising in the Skypeonly condition. A total of six teachers participated in the experiment. The results were normalized by the time length of each session.

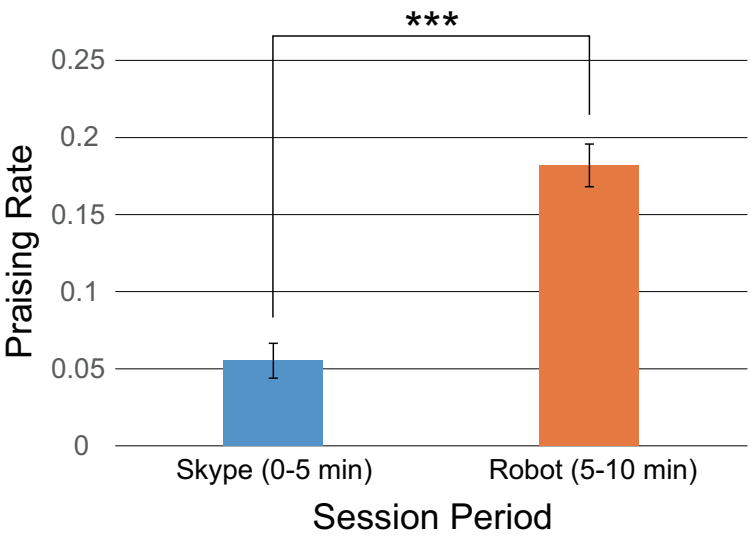

Figure 11: Average accumulated time during which teachers were making positive utterances in the Skype-robot condition. As was the case with the Skype-only condition, six teachers participated in the experiment, and the results were normalized by the time length of each session. The rate was significantly increased in the robot period (Wilcoxon signed-rank test was conducted: $Z(28)=-4.58, p<0.001)$.

some functions of the telepresence robot were observed (Figure 13). They included the movement of the robot base, handling of physical objects using the gripper hand, and gesturing. There also observed another style where the teachers gave instructions about how to move the robot. As shown in Figure 9, teachers often used physical objects to let the children physically participate in some improvised stories that were presented by the teachers. This physical participation, which involved moving their bodies, appeared to have positively affected both the children and teachers.

\subsubsection{Interviewing Children and Teachers}

When each session ended, we interviewed the participant. To participants from the Skype-robot condition group, we asked which situation (Skype only or Skype with an access to the telepresence robot) he/she preferred. To all participants including the ones from the Skype-only condition, we asked if he/she enjoyed the lesson and if he/she became nervous about it. There was one participant

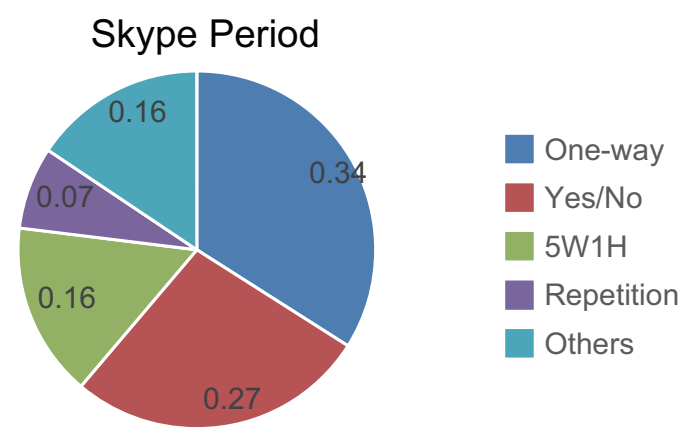

Figure 12: Teaching styles adopted by the six teachers during the Skype period. Rates were calculated on the basis of the amount of time spent utilizing each style. The one-way (unilateral) style and Yes/No style were observed to be dominant.

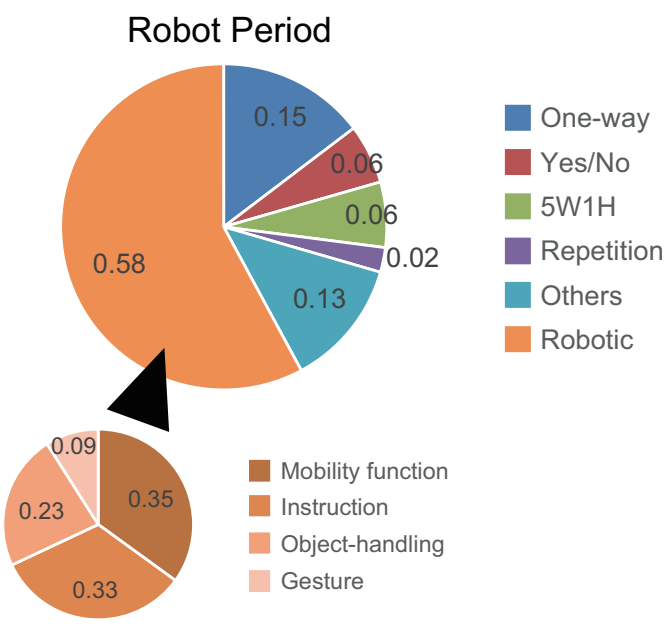

Figure 13: Teaching styles adopted by the six teachers during the robot period. The robotic style included the styles that employed the mobility function, object-handling capability, and gesturing by the robot hand. Using these functions, children were able to physically participate in some stories that were improvised by the teachers.

from the Skype-robot condition whose answer was incomprehensive; thus, we excluded the data from the results. The results show that all the 27 valid participants in the Skype-robot condition preferred the use of the telepresence robot interface. All the participants enjoyed the lesson; however, 10 participants (37\%) in the Skype-robot condition and 9 participants (38\%) in the Skype-only condition said they became nervous during the session.

We also interviewed teachers after the experiment. It was performed by asking them to fill in a survey form, which consisted of two questions and a free description part about the experience of participating in the experiment. The two questions were as follows:

- Q1: Which condition did you feel easier to communicate with children?

- Q2: If you were a classroom teacher, which style do you think you'd like to use?

The results show that five (out of six) teachers chose the robot option for both Q1 and Q2. There was one teacher who chose the 
Skype option, the reason for which was described in the free description part that is reported below (T4).

The list below has some positive comments extracted from the free description part in the survey form given to the teachers:

T1: "Children were active during the robot condition as they felt at ease when interacting with the teacher supported by the robot. The robot condition encouraged children to follow the lesson as they felt excited when they were able to control the robot. In such a situation, the lesson was conveyed in a showcase system instead of just question and answer. Thus, it allowed the teacher to arouse children's interest by connecting the lesson with the movements of the robot. The other advantage was that the robot condition could be used among physically disabled students where the Skype-only condition might fail to do so."

T3: "The participants actively participated in the story-telling activity when the robot was used than when only Skype was used. I think this just confirms that despite most children not understanding English, they enjoy learning through some kind of physical participation. Furthermore, this makes them feel they are part of whatever is happening, and I believe this even helps the children to learn about different things, including a language, faster."

T5: "As a foreigner living in Japan, I get to see this kind of reactions very often, especially in kids, when I try to say hi, and they just freeze, not knowing how to respond, they tend to remain quiet and don't talk. This type of reaction is not only seen in little kids but I have experienced with young people and adults. Japanese people are very kind and willing to help, but when there is a barrier, like language in this case, and they are not prepared for it, they tend to freeze and wait for the situation to be solved by itself. (...) In the robot condition, the communication with the kid was also difficult, but the body language gained a new dimension as it was not just me moving, but I was able to ask the kid to perform an action by showing him, and he could respond. Like, for example, I could ask the kid to follow me to the left, then point to the left, then walk to the left, and so the kid could follow me, even if he didn't understand the word 'left,' he could follow. I felt that the fact that the kid was 'forced' to not be static, made him feel more comfortable and less stressed about the situation of not understanding me. Also, the idea of being playing with a robot controlled at distance seems very exciting for them, and I agree with them, I would also be excited."

Next, listed in below are some negative comments that were also extracted from the survey form. Teacher T4 chose the Skype option for the questions, Q1 and Q2. Teacher T6 chose the robot option for both of the questions.

T4: "In my opinion, the children seem to enjoy the robot condition more because the robot activity makes the process more interesting. On the other hand, as an English teacher, with the robot, it was hard to coordinate story-telling and robot movements. It was hard to make the two go in sync; therefore, I would find myself having difficulty with coming up with a story that matched the robot movement, so I ended up telling the child to close and open their hand or move forward and backward, etc."

T6: "Only stress (with the robot) was that when children didn't break, I didn't know how to operate to stop it. Should I use strength or can I lift it up to make it change direction? If I didn't receive assistance, probably the robot could hit something or someone. I think that the robot can have some sounds when making directions. Like, for example, a little sound (or music) when moving forward so kids can associate the movement with the glove position and learn easier."

In fact, the warning given by teacher T6 is very important from a practical perspective. In this experiment, we dealt with such a safety concern by deploying an experimenter who was in charge of maintaining safety, but in practice, we will be required to incorporate more safety functions into the robot so that this technology is used in realistic scenarios.

\section{DISCUSSIONS}

From the field experiments described in this paper, we confirmed that the issue of children "freezing" did in fact exist in the context of remote communication using a different language and that the use of a telepresence robot system was effective in promoting their communication. It should be noted that both the children and teachers in the remote location had good impressions when using the system for the purpose of remote education.

We explained some results that showed clear advantages of the system such as improvements in the responses of the children. However, to confirm its actual impact on children's learning, a single 5-10 min session is insufficient. However, as discussed in Section 4.3.1, in eight of the participants, we observed that there was rapid learning even within such a short period of time. Considering the interview results, where all of the participants in the Skyperobot condition said that they enjoyed the robot (and most of them wanted to have more lessons), we are optimistic at the prospect of conducting longer or more sessions.

Overall, the experimental results appear to be as expected; however, when we started this research project in 2009, it was not a trivial issue, and many classroom teachers including persons working in professional language schools had high expectations for this project. In this sense, as a case study that was conducted in an actual school environment, the results and knowledge obtained from the experiment are significant.

In this study, we chose a standard video conferencing technology such as Skype as a baseline method and compared it with the telepresence robot system. There are more advanced video conferencing systems that have recently been developed $[14,15,1]$, which offer more interaction capability (e.g., sharing a display) than the conventional video conferencing approach. Although the telepresence robot system still has an advantage because of the ability to realize physical participation in remote environments, there is a need to make further comparisons of these technologies.

It should be noted that multiple elements (factors) are required to form a telepresence robot system. In this study, we focused on only one factor, namely, the operability of the robot provided with the operator by a control interface. However, there are other factors such as the physical appearance of the robot, which was fixed to ensure consistency in our experiment. To provide a more detailed assessment of the effectiveness of a telepresence robot system for remote communication, the roles of these other factors should also be investigated.

It should be noted that the novelty effect may have affected the results that were obtained in this study. The experiment did not completely eliminate this factor, and the results should be evaluated with this limitation in mind.

Regarding the experiment design, we introduced the initial Skype period $(0-5 \mathrm{~min})$ in both conditions so that all participants had the 
same understanding about the nature of a lesson given by a teacher in a remote location. We have been studying child-robot interactions for about 10 years, and we are aware that individual differences often become an issue, particularly in experiments involving young participants. The introduction of a baseline session is a good way to remedy this issue. It was also useful to verify that the two experimental groups were unbiased.

This study is related to the field of embodied learning [16], which utilizes embodied experiences offered by HCI (human-computer interaction) technologies to achieve effective learning. The results presented in this paper (Figure 13) showed more varieties of embodied experiences during the robot period than during the Skype period, which would have resulted in the offering of more embodied learning during the robot period than during the Skype period.

\section{CONCLUSIONS}

This study results suggest that child-operated telepresence robots, which are new to this field, are in fact useful for the purpose of remote education such as teaching foreign languages. We showed that they have the potential to overcome issues encountered during conventional video conferencing, which include the students freezing in front of the monitor, uncertain of what their response should be.

In future, we aim to collaborate with a company that is in the business of teaching English conversation to develop actual lesson plans using the system. Also, we need to investigate the role of other factors in telepresence robots in the context of remote education.

\section{ACKNOWLEDGMENTS}

The authors would like to acknowledge the support provided by the JST PRESTO program and KAKENHI(24119003). We appreciate the cooperation received from the Kasuga Elementary School, Sakura Kindergarten, Higashi Kindergarten, and Ooho Kindergarten during the experiments. We also thank the participating children and their parents, as well as the students of the University of Tsukuba for their cooperation.

\section{REFERENCES}

[1] Svetlana Yarosh, Kori Inkpen, and A.J. Bernheim Brush. Video playdate: toward free play across distance. In Proceedings of the 28th International Conference on Human factors in Computing Systems, pages 1251-1260, Atlanta, USA, 2010. ACM.

[2] Sasa Junuzovic, Kori Inkpen, Tom Blank, and Anoop Gupta. Illumishare: sharing any surface. In Proceedings of the 2012 ACM Annual Conference on Human Factors in Computing Systems, pages 1919-1928, Austin, USA, 2012. ACM.
[3] Fumihide Tanaka, Toshimitsu Takahashi, and Masahiko Morita. Tricycle-style operation interface for children to control a telepresence robot. Advanced Robotics, 27(17):1375-1384, 2013.

[4] Anybots, Inc. https://www.anybots.com/

[5] InTouch Health. http://www.intouchhealth.com/

[6] VGo Communications, Inc. http://www.vgocom.com/

[7] Suitable Technologies. https://www.suitabletech.com/

[8] Double Robotics. http://www.doublerobotics.com/

[9] iRobot. http://www.irobot.com/us/

[10] Katherine M. Tsui, Munjal Desai, Holly A. Yanco, and Chris Uhlik. Exploring use cases for telepresence robots. In Proceedings of the 6th International Conference on Human-Robot Interaction (HRI 2011), pages 11-18, Lausanne, Switzerland, 2011. ACM.

[11] Jenay M. Beer and Leila Takayama. Mobile remote presence systems for older adults: acceptance, benefits, and concerns. In Proceedings of the 6th International Conference on Human-Robot Interaction (HRI 2011), pages 19-26, Lausanne, Switzerland, 2011. ACM.

[12] Sangseok Yun, Jongju Shin, Daijin Kim, Chang Gu Kim, Munsang Kim, and Mun-Taek Choi. Engkey: Tele-education robot. In Proceedings of the 3rd International Conference on Social Robotics, pages 142-152, Amsterdam, The Netherlands, 2011. Springer-Verlag.

[13] Fumihide Tanaka, Toshimitsu Takahashi, Shizuko Matsuzoe, Nao Tazawa, and Masahiko Morita. Child-operated telepresence robot: a field trial connecting classrooms between Australia and Japan. In Proceedings of the IEEE/RSJ International Conference on Intelligent Robots and Systems (IROS 2013), pages 5896-5901, Tokyo, Japan, 2013. IEEE.

[14] Svetlana Yarosh, Stephen Cuzzort, Hendrik Müller, and Gregory D. Abowd. Developing a media space for remote synchronous parent-child interaction. In Proceedings of the 8th International Conference on Interaction Design and Children, pages 97-105, Como, Italy, 2009. ACM.

[15] Lyn Pemberton and Marcus Winter. Collaborative augmented reality in schools. In Proceedings of the 9th International Conference on Computer Supported Collaborative Learning, pages 109-111, Rhodes, Greece, 2009. International Society of the Learning Sciences.

[16] David Birchfield, Thomas Ciufo, and Gary Minyard. SMALLab: A mediated platform for education. In Proceedings of the ACM SIGGRAPH 2006 Educators Program, number 33, Boston, USA, 2006. ACM. 